Classification

Physics Abstracts

$84.90-07.50$

\title{
Conception générale des machines asynchrones sans fer en régime permanent
}

\author{
F. Breduge $\left({ }^{1}\right)$, R. Lenz $\left({ }^{1}\right)$, C. Rioux $\left({ }^{2}\right)$, F. Rioux-Damidau $\left({ }^{2}\right)$ et F. Sultanem $\left({ }^{2}\right)$ \\ (1) Alsthom, 3 avenue des Trois Chênes, 90018 Belfort, France \\ $\left({ }^{2}\right)$ Laboratoire d'Electrotechnique des Universités Paris VI et XI, Bât. 214, 91405 Orsay, France
}

(Reçu le 13 juillet 1989, révisé le 14 novembre 1989, accepté le 27 novembre 1989)

\begin{abstract}
Résumé. - On expose les différentes étapes qui ont conduit à la conception des machines asynchrones sans fer. La modélisation du comportement électromagnétique a permis de définir la géométrie conduisant aux meilleures performances (machines longues), l'étude des pertes de préciser la structure du bobinage, la considération des problèmes thermiques de montrer qu'une ventilation par air simple était convenable et celle des aspects mécaniques de proposer des paliers très simples.
\end{abstract}

\begin{abstract}
The different steps which led us to the design of the ironless asynchronous machines are presented. The electromagnetic modeling has permitted to define the geometry giving the best performance (machine having a great length), the study of the losses to precise the structure of the winding, the consideration of the thermal problems to show that a ventilation by simple use of air was appropriate and that of mechanical aspects to propose very simple bearings.
\end{abstract}

\section{Introduction.}

La réalisation de machines puissantes (qqs $\mathrm{MW}$ ) à vitesses à la fois élevées (qqs $10^{4} \mathrm{t} / \mathrm{min}$ ) et variables, est un problème d'électrotechnique difficile pour des raisons essentiellement mécaniques. En effet, la densité de forces de volume des machines électriques est toujours faible (en comparaison par exemple avec celle des moteurs thermiques). C'est pourquoi il faut mettre en jeu d'importantes masses, se déplaçant à des vitesses élevées. Dans les machines tournantes, il en résulte deux difficultés essentielles : des contraintes mécaniques intenses dues aux forces centrifuges et de nombreuses vitesses critiques méca-

Les machines à courant continu étant à éliminer pour des questions de réalisation du collecteur, deux solutions classiques restent envisageables, mais elles présentent l'une et l'autre un certain nombre de problèmes. Tout d'abord la machine synchrone exige la réalisation de circuits bobinés tournant à grande vitesse, ce qui entraîne une limitation de la vitesse périphérique du rotor à une valeur de l'ordre de 200 à $250 \mathrm{~m} / \mathrm{s}$. Pour ce qui est des solutions asynchrones, la réalisation du rotor est certes plus aisée (cage d'écureuil) mais les vitesses limites restent du même ordre. Dans les deux cas, les stators sont semblables, mettant en œuvre la technique consistant à distribuer les conducteurs dans un système dents/encoches. Lors de la rotation du rotor, il apparaît ainsi au niveau de ce dernier des champs de haute fréquence engendrant dans le fer des pertes superficielles très élevées (dites pertes de denture). Les difficultés que l'on vient de décrire restent du même type sur les machines homopolaires rapides, bien que ces dernières ne comportent pas de matériaux conducteurs sur les parties tournantes.

Dans un tel contexte, il nous a semblé intéressant d'examiner une autre voie : celle des machines sans fer. Ces machines présentent tout d'abord un avan$\mathrm{u}$ : eur puissance $\mathrm{n}$ es pas $1 \mathrm{~m}$ ee, puisqu'elles ne présentent aucun phénomène de saturation (c'est cette qualité qui a incité à les étudier pour les régimes impulsionnels $[1,2]$, où la mise en œuvre de fortes puissances massiques est indispensable). Mais elles ont aussi un grave inconvénient : leur rendement ne devient acceptable que si le produit des vitesses linéaires par les dimensions est élevé (en pratique, ces machines sont inutilisables aux basses puissances ou aux faibles vitesses). D'autre part, sous leur forme asynchrone, le rotor est extrêmement simple (il est constitué par un 
simple cylindre conducteur massif), tandis que les pertes de denture ont disparu.

Pour les régimes permanents, on est séduit par les deux derniers points : la simplicité et l'absence des pertes de denture. De plus, la stabilité électromagnétique du rotor, intrinsèque à ce type de machine, a permis d'innover en matière de suspension : placé sur des paliers flottants, même très éloignés, le rotor ne présente aucune vitesse critique mécanique dans une très large plage de fonctionnement. Enfin, il s'est révélé à l'étude que des moyens de refroidissement par simple ventilation étaient possibles en conservant des densités de puissance assez élevées $[3,4]$. Ce sont les principes de construction et les divers problèmes rencontrés dans la modélisation et la conception de ces machines, qui vont être exposés ci-après.

\section{Structure générale.}

Une machine asynchrone sans fer typique est représentée schématiquement sur la figure 1.

Le rotor (1) en alliage d'aluminium AU2GN massif est supporté par des paliers (4) (roulements à billes), associés à une suspension souple (5).

Le stator (2) est constitué par un bobinage triphasé quasi-classique à «têtes couchées" de manière à présenter une forme cylindrique sur toute sa longueur. Il est alimenté par un onduleur connecté aux différentes phases du bobinage.

Le refroidissement s'effectue par une circulation forcée d'air (entrée-sortie (6)-(7)). Ce dernier circule dans le bobinage " aéré » et lèche parallèlement la périphérie du rotor.

Un prototype a été construit et expérimenté. Le rotor avait un diamètre de $16 \mathrm{~cm}$ et une longueur de $1 \mathrm{~m}$.
3. Caractéristiques dimensionnelles générales des machines sans fer.

Les machines sans fer, qui ne présentent aucun phénomène de saturation, sont régies par des lois d'homothétie sensiblement différentes de celles des machines classiques. En effet, les machines classiques sont conçues pour fonctionner dans des conditions proches des limites de saturation (voire à la saturation à proprement parler) ; ceci a pour effet de fixer la valeur de l'induction magnétique moyenne $B$ à la moitié de l'inductance de saturation $B_{\mathrm{S}}$. En ce qui concerne les machines sans fer, $B$ est proportionnel au courant.

Considérons donc un ensemble de machines homothétiques différenciées par la donnée d'une de leurs dimensions, par exemple le rayon $R$ du rotor. La puissance d'une machine est telle que :

$$
P \sim\left(B_{j}\right)(\Omega R) R^{3}
$$

où :

$B$ est l'induction

$j$ est la densité de courant dans les parties conductrices du rotor

$\Omega$ est la vitesse angulaire du rotor

$R$ est le rayon du rotor

(Le volume des conducteurs du rotor est proportionnel à $R^{3}$, car le rapport de l'épaisseur de peau au rayon du rotor est le même au glissement donnant la puissance maximale, quelles que soient les dimensions).

La puissance Joule dissipée dans le rotor vaut :

$$
P_{\mathrm{J}} \sim \frac{1}{\sigma} j^{2} R^{3}
$$

où $\sigma$ et la conductivité des matériaux.

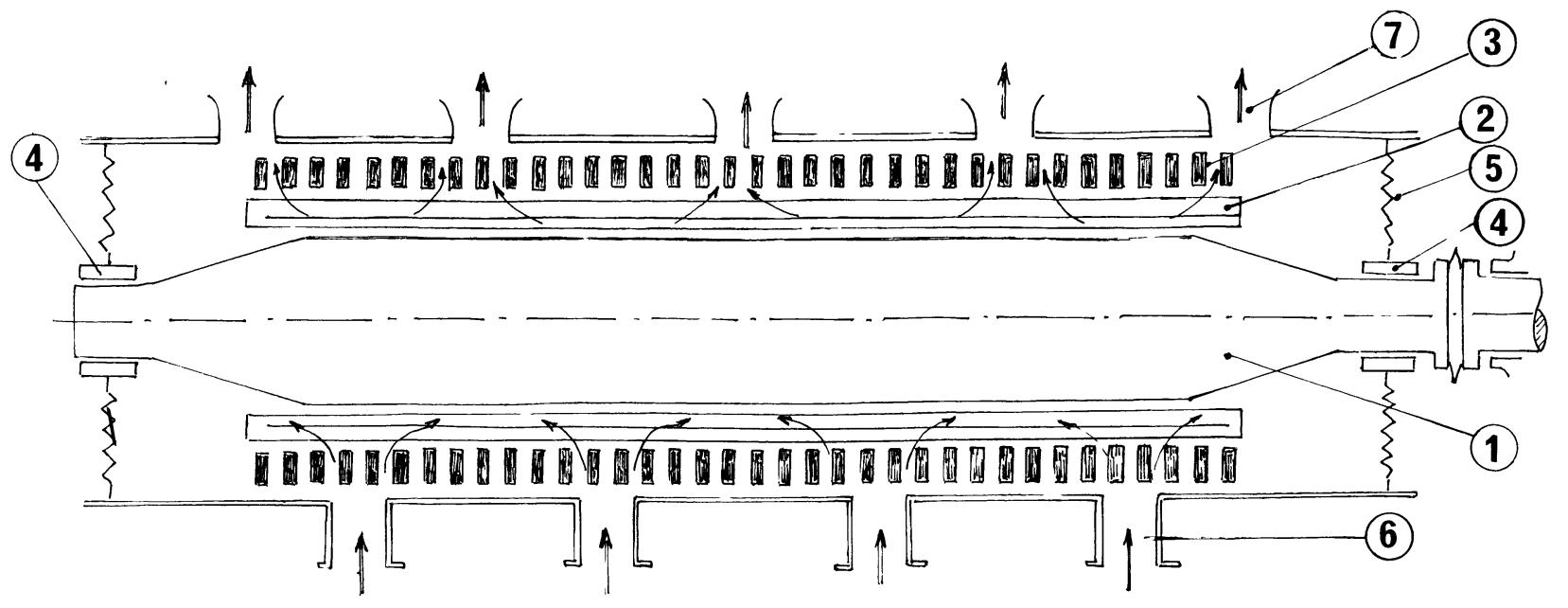

Fig. 1. - Schéma de principe d'une machine asynchrone sans fer.

[Scheme of an ironless asynchronous machine.] 
(Pour une machine asynchrone bien conçue, les pertes du stator sont du même ordre que celles du rotor, au sens du présent calcul dimensionnel; de fait, dans le cas expérimenté, les pertes rotor sont plus faibles d'un facteur constant de l'ordre de 5 à 10.)

Si l'on néglige les autres pertes (dont celles de denture), le rendement $\boldsymbol{\eta}$ de la machine est :

$$
\eta=1-\frac{P_{\mathrm{J}}}{P}=1-k \frac{j}{\sigma B \Omega R}
$$

où $k$ est une coefficient de structure issu de la forme géométrique de la machine.

Pour une machine classique, où $B=B_{\mathrm{S}} / 2$, on peut écrire, en introduisant la puissance :

$$
\eta=1-k \frac{4}{\eta B_{\mathrm{S}}^{2}(\Omega R)^{2}} \cdot\left(\frac{P}{R^{3}}\right) \text {. }
$$

On voit ainsi que le rendement $\eta$ diminue avec la puissance volumique $P / R^{3}$ et croît avec la vitesse linéaire $\Omega R$.

Pour une machine sans fer, l'induction créée par le stator est de la forme :

$$
B \sim \mu_{0} j R .
$$

(On rappelle que les densités de courant au stator et au rotor ne sont pas très différentes.)

Le rendement devient alors :

$$
\eta=1-k \frac{1}{\mu_{0} \sigma(\Omega R) R} .
$$

Il en résulte que le rendement électrique est indépendant de la puissance et croît avec la vitesse linéaire et les dimensions.
Au point de vue pratique, le coefficient de géométrie $k$ introduit ici est assez différent pour les machines classiques et sans fer. Lorsqu'on tient compte de tous les éléments, on peut dire, grossomodo, que le rendement des machines sans fer devient de l'ordre de celui des machines classiques de même puissance lorsque le champ magnétique en leur intérieur est voisin de $B_{\mathrm{S}} / 10$.

L'intérêt de la machine sans fer vis-à-vis des formes classiques croît lorsque la puissance augmente [3].

\section{Caractéristiques électromagnétiques.}

Elles ont été calculées à partir de la modélisation tridimensionnelle de ces machines pour un fonctionnement en régime permanent.

4.1 Modélisation. - Aucun phénomène de saturation n'apparaissant dans ces machines, ces dernières ont un comportement linéaire. De plus, la machine toute entière, hormis le bobinage, est cylindrique et de révolution.

Cette situation très favorable nous a incité à utiliser directement une modélisation par séries de Fourier sur les trois variables $t, \theta$ et $z$. Sur ces trois variables, seule $\theta$ conduit naturellement à un spectre discret. Pour un régime permanent, il en est de même pour $t$. En ce qui concerne $z$, le traitement direct implique l'introduction d'une intégrale de Fourier. Mais, pour faciliter le traitement numérique, on a discrétisé artificiellement le spectre sur $z$ en remplaçant (cf. Fig. 2a) la machine étudiée par l'ensemble d'une infinité de machines identiques régulièrement espacées le long de l'axe $\mathrm{O} z$ et

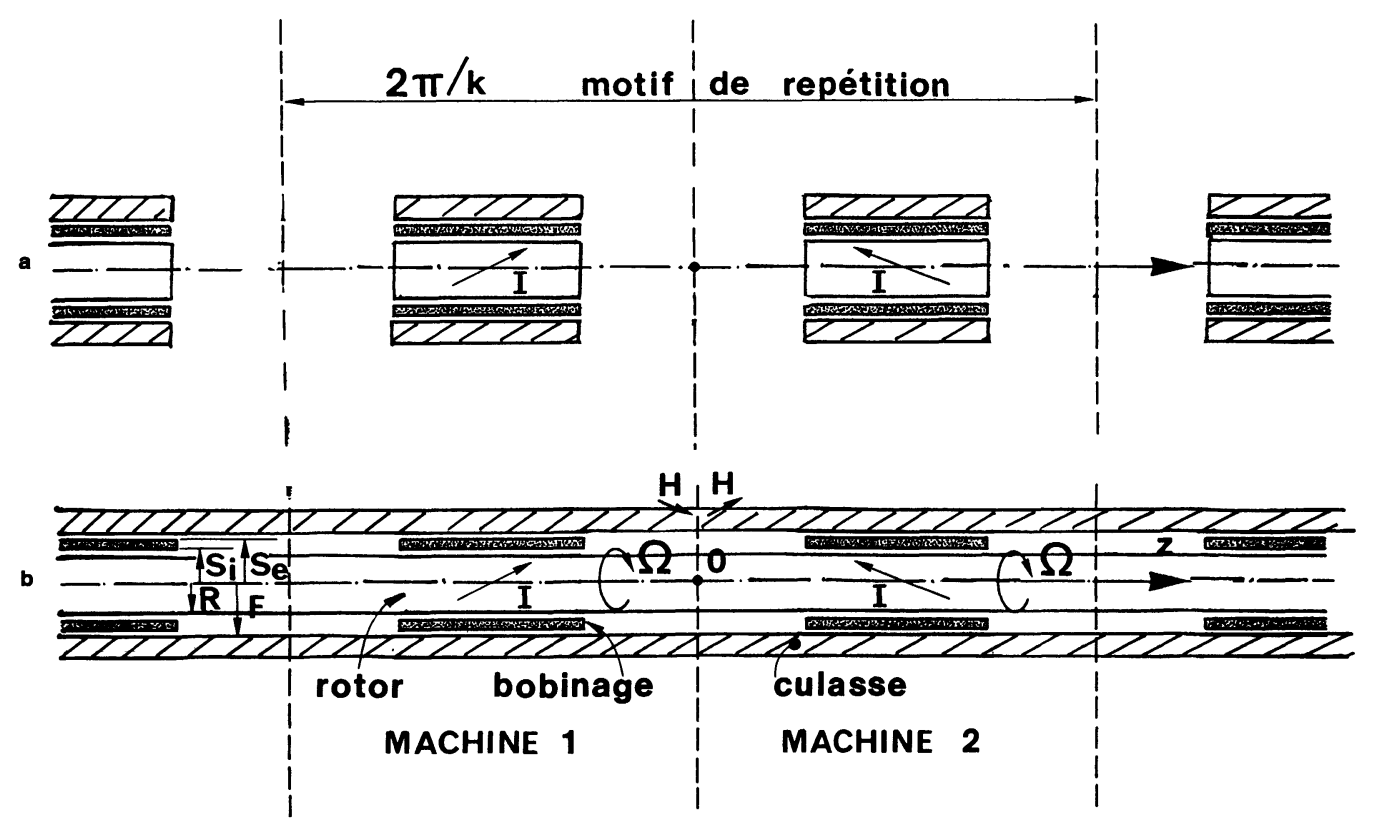

Fig. 2. - Schéma du système modélisé, avec rotor et culasse réels (a) et rotor et culasse de longueur «infinie » (b). [Scheme of the modeled system, with the real rotor and yoke (a) and with the «infinitely» long rotor and yoke (b).] 
suffisamment éloignées l'une de l'autre pour ne pas interagir entre elles. Toutes les décompositions en séries de Fourier ont évidemment la même forme générale ; à titre d'exemple, le champ magnétique s'écrit :

$$
\mathbf{H}(\rho, \theta, z, t)=\sum_{n \ell q \in \mathbb{Z}^{3}} H_{n \ell q}(\rho) \mathrm{e}^{i(n \omega t+\ell \theta+q k z)}
$$

où :

$\omega$ est la pulsation du courant d'alimentation, qui est périodique mais de forme quelconque

$2 \pi / k$ le pas en $z$ du système

$\mathbb{Z}$ est l'ensemble des entiers relatifs.

En reportant ces décompositions dans les équations de Maxwell, on obtient les équations différentielles auxquelles obéissent les coefficients $H_{n \ell q}(\rho)$, et que l'on a alors résolues.

4.2 SPeCTRE DES IMPÉdANCES. - En régime linéaire et en fonctionnement permanent, les caractéristiques électriques de la machine sont entièrement définies par le spectre de ses impédances aux différentes fréquences.

La pulsion fondamentale étant $\omega$, on peut écrire parallèlement la tension $\mathbb{V}(t)$ et le courant $\mathbb{l}(t)$ sous les formes spectrales :

$$
\begin{aligned}
& \mathbb{V}(t)=\sum V_{n} \mathrm{e}^{i n \omega t} \\
& \mathbb{J}(t)=\sum J_{n} \mathrm{e}^{i n \omega t} .
\end{aligned}
$$

Les équations de Maxwell permettent de relier facilement les valeurs de $V_{n}$ et $J_{n}$ en fonction des caractéristiques géométriques du bobinage et des champs magnétiques au niveau de ce dernier (en particulier, cette liaison dépend du glissement du rotor).

On peut alors écrire :

$$
V_{n}=Z_{n} I_{n}
$$

et déterminer le spectre des impédances $Z_{n}$. Pour des raisons pratiques de traitement, on a posé plus précisément :

$$
Z_{n}=n \omega\left(L_{n \mathrm{r}}+j L_{n \mathrm{i}}\right) .
$$

On en tire alors les expressions des puissances actives et réactives :

$$
\begin{aligned}
& P_{\mathrm{a}}=2 m p \sum_{n \in \mathbb{N}} n \omega L_{n \mathrm{r}}\left|J_{n}\right|^{2-} \\
& P_{\mathrm{r}}=2 m p \sum_{n \in \mathbb{N}} n \omega L_{n \mathrm{i}}\left|J_{n}\right|^{2}
\end{aligned}
$$

où :

$p$ est le nombre de paires de pôles $m$ est le nombre de plages de phases $\mathbb{N}$ est l'ensemble des entiers naturels.
4.3 Mise en eeUVRe du CAlcul. - Au point de vue pratique, on a procédé pour le calcul des champs en deux temps. Dans un premier temps [5], on a supposé que le rotor et la ceinture magnétique étaient infiniment longs (voir Fig. 2), ce qui s'exprime mathématiquement dans la décomposition en écrivant que le rotor et la ceinture ont une longueur égale au demi-pas de la décomposition en $z$ (soit $\pi / k$ ). Dans ce cas, les équations résultantes sont de type Bessel et on obtient une solution analytique facilement programmable, conduisant de surcroît à des temps de calcul extrêmement courts (car le nombre d'harmoniques ainsi nécessaires est très faible).

Dans un deuxième temps, on s'est livré à des calculs plus élaborés où les longueurs finies du rotor et du stator ont été prises en compte. Plus précisément, on a tout d'abord étudié de manière détaillée l'effet de longueur finie du rotor dans une ceinture illimitée [6]. On a constaté que l'approximation du rotor infini était excellente :

- pour $L / 2 R \simeq 1$ (machine " carrée »), l'erreur en moyenne n'excède guère $10 \%$;

- pour $L / 2 R \simeq 1,6$, l'erreur descend à $2 \%$.

Or, les machines réelles doivent avoir des rapports $L / 2 R$ grands parce que le rendement est alors plus élevé. Pour le prototype construit où $L / 2 R=6$, l'erreur est tout à fait négligeable. En considérant une ceinture courte avec un rotor infini, l'erreur est moindre encore [7].

Cette modélisation a été ensuite testée sur un dispositif statique construit dans ce but [8] (il reproduisait le fonctionnement réel d'une machine tournante par un choix convenable de la résistivité du rotor). L'expérience a montré que la détermination des grandeurs énergétiques (couple, puissances) était obtenue de façon tout à fait correcte avec un faible nombre d'harmoniques (moins de 10) alors que la description détaillée des champs en demandait beaucoup plus (50 à 100).

4.4 Performances. - Les impédances $Z_{n}$ ont des propriétés très intéressantes qui permettent de les déterminer toutes à partir d'un petit nombre d'entre elles [9]. Pour une machine bipolaire $(p=1)$ à 12 bobines, on a (pour $n \geqslant 5$ et $k \in \mathbb{N}$ ) :

si $n=12 k \pm 1: L_{n \mathrm{i}}=\alpha_{1} / \sqrt{n \Omega} ; L_{n \mathrm{r}}=\beta_{1}$

si $n=12 k \pm 5: L_{n \mathrm{i}}=\alpha_{5} / \sqrt{n} ; \quad L_{n \mathrm{r}}=\beta_{5}$

(les autres impédances n'interviennent pas, car les harmoniques du courant d'ordre multiple de 2 ou 3 sont nuls)

avec :

$$
\begin{aligned}
& L_{n \mathrm{i}} \gg L_{n \mathrm{r}} \\
& L_{1} \gg L_{n}(n \neq 1) .
\end{aligned}
$$

Ainsi, les puissances actives et réactives de ces machines sont essentiellement dues au fondamental. Les performances s'accroissent lorsque le rapport 

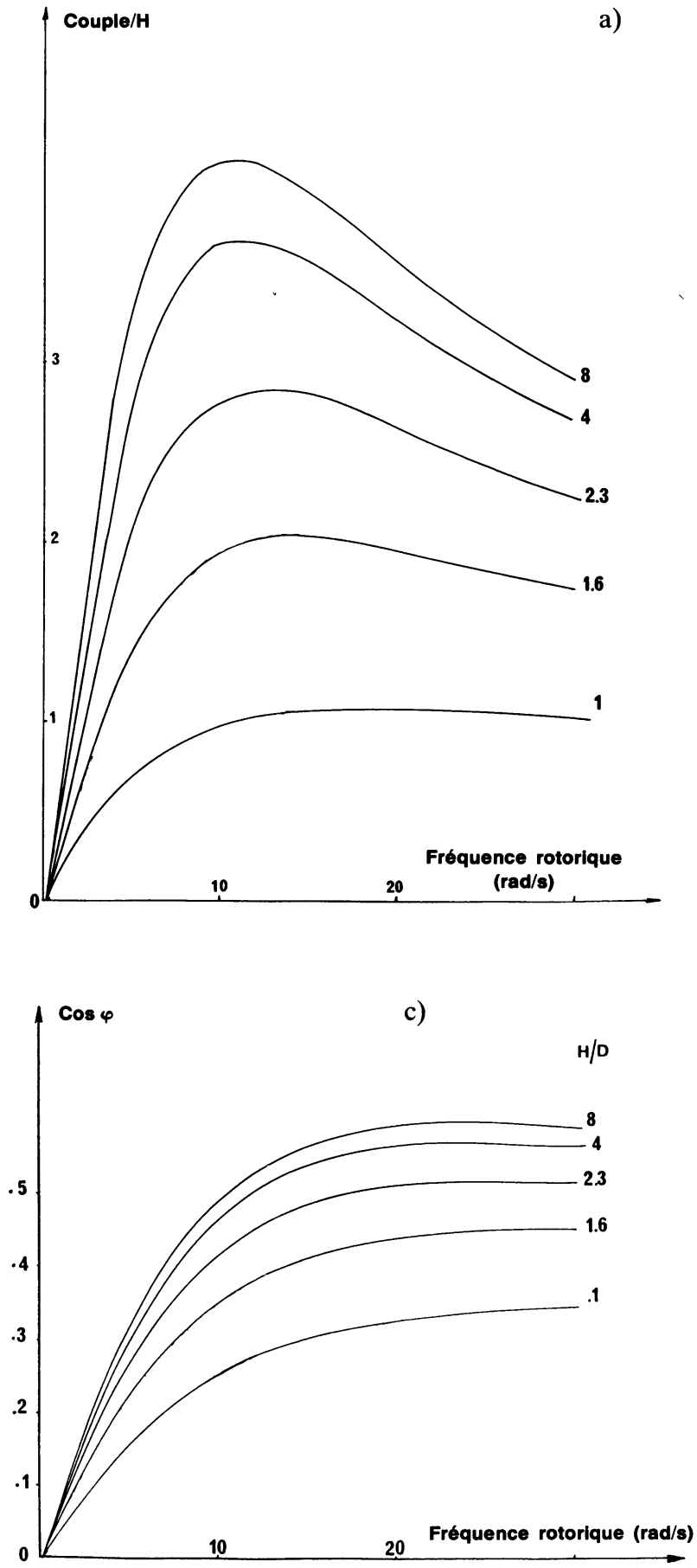

Fig. 3. - Couple par unité de longueur (a), puissance réactive (b) et facteur de puissance (c) pour diverses

dimensions identiques.

[Torque per unit length (a), reactive power (b) and power factor (c) for several machines having different length and equal other dimensions.]

longueur/diamètre $H / 2 R$ croît (cf. Fig. 3). En pratique, cependant, le gain des performances par unité de longueur ne croît que faiblement au-delà de $H / 2 R=4$. Le facteur de puissance est assez faible, de l'ordre de 0,5 à 0,6 .

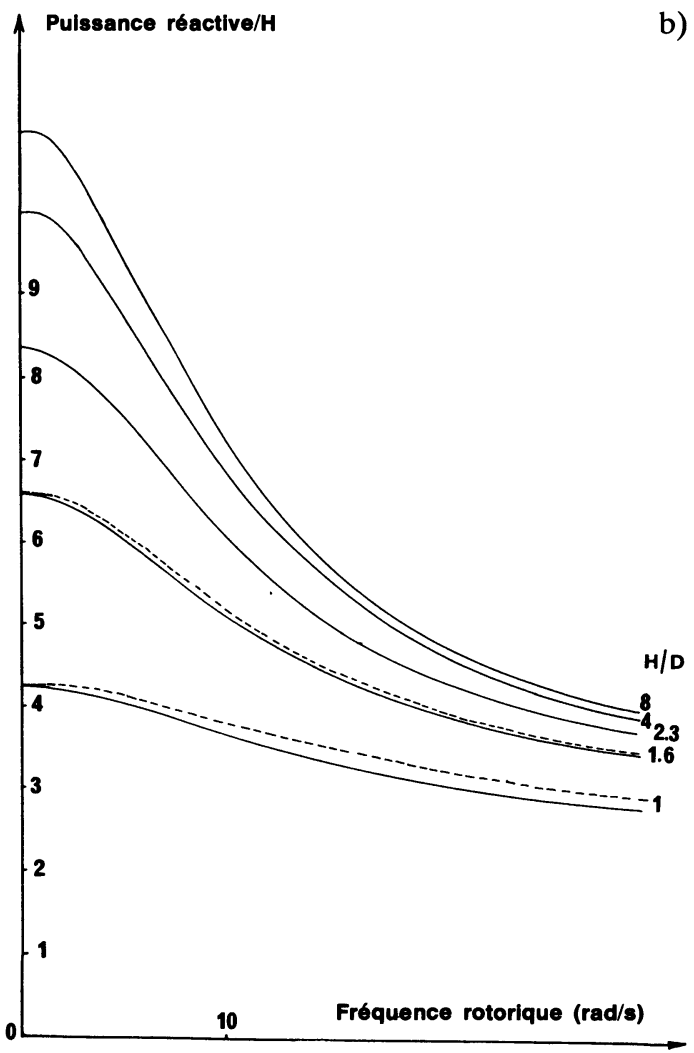

b)

Le bobinage peut être hélicoïdal comme dans le prototype ou être parallèle à $\mathrm{O} z$ au milieu de la machine sur une longueur $H_{\mathrm{d}}$ et incliné seulement sur les longueurs $\left(H-H_{\mathrm{d}}\right) / 2$ des extrémités (bobinages « hexagonal ») [10]. Cette dernière configuration accroît le rendement (d'environ $20 \%$ pour la valeur optimale $H_{\mathrm{d}} / H \sim 0,5$ ) et ne modifie pas le facteur de puissance. On préfère cette solution de "bobinage couché " à la forme "classique » qui serait dans ce cas constituée par des conducteurs droits et des têtes de bobines conventionnelles et qui présenteraient des $\cos \varphi$ nettement inférieurs.

\section{Pertes.}

5.1 Pertes Rotoriques. Structures du bobiNAGE. - Outre les pertes rotoriques inévitables iees au onctionnement async rone proprement it (dues au fondamental et proportionnelles au glissement), il apparaît au niveau du rotor des phénomènes de freinage et de dissipation provoqués par les harmoniques. Pour éliminer les pertes qui leur sont associées, il est donc nécessaire d'amoindrir dans toute la mesure du possible la présence d'harmoniques au niveau du stator. Dans le cas d'un bobinage triphasé simple habituel, les harmoniques « inutiles » entraînent des pertes supplémentaires relativement importantes [11].

Pour réduire ces pertes à une valeur acceptable, 
on a mis en œuvre dans le prototype un bobinage à douze phases, matérialisé par un double bobinage triphasé décalé de $30^{\circ}$. Pour des questions de refroidissement qui seront examinées plus loin, le bobinage est très « aéré », c'est-à-dire que chaque barre du bobinage est séparée de la suivante dans le sens azimutal par un vide de dimension comparable à l'épaisseur de la barre. Cette inhomogénéité de la répartition des conducteurs engendre une distribution locale de champ à haute fréquence spatiale correspondant à un champ homopolaire à 36 paires de pôles mais de très faible amplitude au niveau du rotor. Les pertes qui en résultent sont négligeables (elles sont incomparablement plus faibles que les pertes de denture habituelles des machines comportant des matériaux ferromagnétiques). Cette structure serait bien adaptée à une machine industrielle.

\subsection{PERTES PAR COURANTS DE FOUCAULT STATORI-} QUES. - Contrairement à ce qui se passe dans les machines classiques, les conducteurs du bobinage sont soumis à la totalité du champ d'induction. Il doit en résulter a priori des pertes par courants de Foucault importantes. Comme il est bien connu, ces pertes sont proportionnelles au produit des carrés du champ, de la fréquence et des dimensions transversales des conducteurs $\left(B^{2} \omega^{2} e^{2}\right)$. Dans le cas présent, la fréquence est élevée mais le champ est faible ; il n'est donc pas nécessaire de diviser extrêmement les conducteurs. Ces pertes ont été calculées de manière précise, à partir des champs déterminés par la modélisation décrite plus haut [12]. En pratique, l'emploi de barres Roebell à 17 brins au niveau du prototype est amplement suffisant; dans une machine effective, les pertes par courants de Foucault n'excéderaient pas quelques \% des pertes Joule directes.

5.3 PERTES DANS LA CULASSE. - On remarquera tout d'abord que le faible champ dans l'entrefer ne demande qu'une culasse de retour de petit volume. Pour faciliter sa construction et la circulation de l'air, on peut constituer cette culasse par une série d'anneaux (voir Fig. 1). Comme la décroissance axiale des densités moyennes de courants est lente, les lignes de champ magnétique s'écartent peu des plans normaux à l'axe. Par suite, les lignes de champ restent toujours sensiblement parallèles au plan des tôles, ce qui minimise encore les pertes. Ces dernières sont finalement tout à fait négligeables.

\section{Les aspects thermiques.}

Les machines sans fer ne présentant aucun phénomène de saturation au niveau du rotor et du stator, leur puissance maximale instantanée n'est guère limitée que par la résistance mécanique du rotor. Il en résulte que la puissance moyenne possible ne dépend en pratique que des procédés d'évacuation de la chaleur dégagée.

Dans le cas du prototype, on a choisi un mode de refroidissement très simple, une circulation d'air. Les deux principales sources de dégagement de la chaleur sont le rotor et le stator, la plus importante étant cette dernière. Le refroidissement du bobinage s'effectue par injection longitudinale d'air dans les espaces séparant les barres conductrices. Cet air est ensuite dérivé vers la surface du rotor, qui est ainsi léchée. On remarquera que la surface de refroidissement du rotor est beaucoup plus petite que celle du stator, mais elle est suffisante car la chaleur dégagée par le rotor est relativement faible (environ dix fois plus petite).

\section{Les aspects mécaniques.}

Une caractéristique fondamentale des machines asynchrones sans fer est leur stabilité électromagnétique transversale par rapport à leur axe, car les effets d'écran d'un rotor en rotation ont pour conséquence, vis-à-vis d'un observateur extérieur, de lui donner le comportement d'un matériau diamagnétique (les lignes de champ sont repoussées vers l'extérieur). Or, un matériau diamagnétique est naturellement attiré par les régions de champ faible, c'est-à-dire vers l'axe de la machine. Plus précisément, le rotor est en équilibre indifférent dans un champ bipolaire parfait et strictement stable pour les champs de polarité plus élevée.

Cette situation a entraîné la conception d'une machine aux formes tout à fait inhabituelles. En effet, nous avons vu plus haut que, pour améliorer les performances électromagnétiques il est essentiel de mettre en œuvre un rapport longueur/diamètre du rotor grand. Or, il est bien connu que si un rotor long est guidé par des paliers rigides (cas habituel), une haute vitesse de rotation ne peut être obtenue qu'en franchissant de nombreuses vitesses critiques mécaniques.

Grâce à la stabilité électromagnétique du rotor, on peut tourner cette difficulté importante en choisissant un mode de suspension peu courant : les paliers peuvent en effet être montés sur une suspension souple à base de blocs de caoutchouc. Il en résulte que les premières vitesses critiques mécaniques se trouvent situées dans la zone des basses vitesses où elles n'apportent en pratique aucun inconvénient.

\section{Conclusion.}

La construction de machines cylindriques asynchrones sans fer, ayant un rapport longueur/diamètre $\geqslant 4$ et montées sur des paliers souples, permet de réaliser des unités ayant une puissance nominale élevée (quelques MW), fonctionnant à grande vitesse avec un bon rendement, mais présentant un facteur de puissance relativement faible. L'amélioration de 
ce facteur de puissance peut être obtenue par l'introduction d'un noyau magnétique [13], mais au prix d'une réduction de la vitesse périphérique.
Le développement de ce type de machine, avec ou sans noyau, n'est pas encore effectué au point de vue industriel.

\section{Bibliographie}

[1] Rioux C., Recherche critique d'une forme d'électrotechnique adaptée aux grandes puissances, Thèse de Doctorat d'Etat, Paris (1964).

[2] Rioux C., Sultanem F., A solid rotor iron-free asynchronous machine, J. Appl. Phys. 50 (1979) 574-581.

[3] Rioux C., Bleiss C. A., Rioux-Damidau F., Sultanem F., Machines sans fer : éléments de comparaison entre calculs et essais sur maquette, Rev. Gen. Elec. 10 (1984) 619-626.

[4] Dhers J., Rioux C., Rioux-Damidau F., SultaNEM F., Characteristics and interest of iron-free asynchronous generators and motors, Congrès AEI, Turin (1986).

[5] Rioux-Damidau F., Calcul semi-tridimensionnel des champs et des courants dans les machines cylindriques asynchrones sans fer, Revue Phys. Appl. 18 (1983) 13-127.

[6] Rioux-Damidau F., Modélisation des machines cylindriques asynchrones sans fer. Prise en compte de la longueur finie du rotor, Revue Phys. Appl. 20 (1985) 235-242.

[7] Bandelier B., RiouX-Damidau F., Modélisation des machines cylindriques asynchrones sans fer. Prise en compte de la longueur finie de la culasse, Revue Phys. Appl. 21 (1986) 327-337.
[8] Bleiss C. A., Letellier J. L., Rioux C., RiouxDAmidau F., Détermination expérimentale des caractéristiques des machines sans fer et comparaison avec une modélisation pseudo-tridimensionnelle, Revue Phys. Appl. 20 (1985) 583-587.

[9] Rioux-Damidau F., Alame N., Computation of the impedances of the iron-free asynchronous machines, Elect. Machines Pow. Syst. 11 (1986) 137-146.

[10] BANDELIER B., RiOUX-DAMIDAU F., Influence of the structure of windings in iron-free asynchronous machines, Elect. Machines Pow. Syst. 12 (1987) 233-242.

[11] Bleiss C. A., Contribution à l'étude de l'alimentation des machines asynchrones à rotor massif non magnétique, Thèse de doctorat d'état - Université Paris VI (1981).

[12] Gueraud A., Bandelier B., Rioux-Damidau F., Calcul des pertes par courants induits dans les conducteurs statoriques des machines asynchrones sans fer, Revue Phys. Appl. 24 (1989) 659669.

[13] Rioux-Damidau F., Rioux C., Gueraud A., Machines asynchrones à rotor massif composite, Revue Phys. Appl. 24 (1989) 1039-1047. 